\title{
Sensitivity and Generalization of a Neural Network for Estimating Left Atrial Fibrotic Volume Fractions from the 12-lead ECG
}

Claudia Nagel, Institute of Biomedical Engineering, Karlsruhe Institute of Technology, Kaiserstr. 12, 76131 Karlsruhe, Germany,publications@ibt.kit.edu

Olaf Dössel, Axel Loewe, Institute of Biomedical Engineering, Karlsruhe Institute of Technology, Kaiserstr. 12, 76131

Karlsruhe, Germany, publications@ibt.kit.edu

Features extracted from $P$ waves of the 12-lead electrocardiogram (ECG) have proven valuable for non-invasively estimating the left atrial fibrotic volume fraction associated with the arrhythmogenesis of atrial fibrillation. However, feature extraction in the clinical context is prone to errors and oftentimes yields unreliable results in the presence of noise. This leads to inaccurate input values provided to machine learning algorithms tailored at estimating the amount of atrial fibrosis with clinical ECGs. Another important aspect for clinical translation is the network's generalization ability regarding new ECGs.

To quantify the network's sensitivity to inaccurately extracted P wave features, we added Gaussian noise to the features extracted from 540,000 simulated ECGs consisting of P wave duration, dispersion, terminal force in lead V1, peak-topeak amplitudes, and additionally thoracic and atrial volumes. For assessing generalization, we evaluated the network performance for train-validation-test splits divided such that ECGs simulated with the same atria or torso geometry only belonged to either the training and validation or the test set.

The RMSE of the network increased the most in case of noisy torso volumes and $\mathrm{P}$ wave durations. Large generalization errors with RMSE difference between training and test set of more than $2 \%$ fibrotic volume fraction only occurred if very high or low atria and torso volumes were left out during training.

Our results suggest that $\mathrm{P}$ wave duration and thoracic volume are features that have to be measured accurately if employed for estimating atrial fibrosis with a neural network. Furthermore, our method is capable of generalizing well to ECGs simulated with anatomical models excluded during training and thus meets an important requirement for clinical translation. 


\section{Classification of Bundle Branch Blocks with QRS Templates Extracted from 12-lead ECGs}

Hannes Welle, Institute of Biomedical Engineering, Karlsruhe Institute of Technology (KIT), Karlsruhe, Germany, publications@ibt.kit.edu Claudia Nagel, Institute of Biomedical Engineering, Karlsruhe Institute of Technology (KIT), Karlsruhe, Germany Axel Loewe, Institute of Biomedical Engineering, Karlsruhe Institute of Technology (KIT), Karlsruhe, Germany Ralf Mikut, Institute for Automation and Applied Informatics, Karlsruhe Institute of Technology (KIT), Karlsruhe, Germany

Olaf Dössel, Institute of Biomedical Engineering, Karlsruhe Institute of Technology (KIT), Karlsruhe, Germany

\section{Introduction}

The digitalization of healthcare leads to an increased demand for and feasibility of automatic data-driven diagnostic tools. Being non-invasive, cheap and widely available, the 12-lead electrocardiogram (ECG) is a standard method to assess cardiac function.

\section{Methods}

We evaluated the performance of machine learning-based classification of healthy and bundle branch block 12-lead ECGs from three publicly available datasets comprising a total of 11,705 signals. For each lead of the ECG signal, a representative QRS-complex template was extracted automatically. Principal component analysis (PCA) was applied to the concatenated, normalized and rescaled QRS signals to reduce their dimensionality. Multilayer perceptron and supportvector machine classifiers were trained using the principal components of weighted and non-weighted QRS template signals as input data.

\section{Results}

Classifiers achieved F1 scores between 0.92 and 0.96 on the test set for different input configurations. The anomaly-based rescaling slightly improved the performance of the classifiers. Neither class-wise PCA for feature extraction nor adding information on sex, gender and electrical heart axis to the input data yielded considerable improvement of the F1 scores.

\section{Conclusion}

The achieved classification accuracy is similar to deep learning classifier performances and should generalize robustly to other ECG datasets. Our results suggest that this simple and well interpretable approach based on morphological signal characteristics is suitable for automatically and non-invasively identifying bundle branch block pathologies in clinical or smart electronics contexts. 


\section{Finding an optimal dictionary of different wavelet types using sparse modeling to denoise ECG signal}

Fars Samann, Institut für Biomedizinische Technik (IBMT), FB Life Science Engineering (LSE), Technische Hochschule Mittelhessen (THM) - University of Applied Sciences, Gießen, Germany,

fars.esmat.fathel.samann@1se.thm.de, and, Biomedical Engineering Department, College of Engineering, University of Duhok, Duhok, Kurdistan Region, Iraq

Thomas Schanze, Institut für Biomedizinische Technik (IBMT), FB Life Science Engineering (LSE), Technische Hochschule Mittelhessen (THM) - University of Applied Sciences, Gießen, Germany, thomas.schanze@1se.thm.de

\section{Introduction}

Denoising electrocardiogram (ECG) signal is significantly important for accurate diagnosing of heart diseases. Several sparse denoising models (SDM) were developed for denoising biomedical signals. However, to achieve adequate SDM, it is critical to establish (build) a dictionary consisting of atoms which sufficiently represent the target signal. The challenge of finding an optimal dictionary of different wavelet types using SDM to denoise ECG signal is investigated in this work.

\section{Methods}

Sparse modeling often reconstructs a signal with few atoms from a pre-defined dictionary. We constructed three wavelet dictionaries which consist of $N$ sub-dictionaries of the same wavelet type of level 3: symN, dbN, coifN. Then, QRS complex aligned 200 ECG segments from CPSC database (segment length 396 samples and $f_{\text {sampling }}=500 \mathrm{~Hz}$ ) from 20 healthy persons are applied to matching pursuit SDM with nonzero coefficients equal to ECG segment length. This is to ensure the full reconstruction of the clean ECG segments. For each sub-dictionary, the required number of atoms and the correlation between original signal and these atoms' waveform are calculated. The sub-dictionaries with high correlation and few atoms are considered to construct the optimal dictionary. For evaluation corrupted ECG segments with simulated white noise of different input $S N R_{i}(10,20,30 \mathrm{~dB})$ are generated. The performance of the main three dictionaries and the optimal dictionary in denoising ECG segments are evaluated in terms of number of atoms and the improvement of $S N R_{\text {imp }}$. The Akaike's information criterion is used to find the optimal number of atoms.

\section{Results}

The results obtained for $S N R_{i}=30 \mathrm{~dB}$ show that the optimal dictionary has $S N R_{\text {imp }}=1.18 \mathrm{~dB}$ and only required 93 atoms, while symN, dbN, coifN dictionaries have $S N R_{\text {imp }}=0.033,0.044,-0.075 d B$ and 113, 116, 133 atoms respectively.

\section{Conclusion}

The proposed method succeeds in finding strong signal-correlated and likely sparse dictionary to denoise a specific signal. 


\section{Robust and body-specific determination of EASI matrices for the cal- culation of Einthoven and Goldberger leads from EASI leads}

Melanie Holderith, Institute for Biomedical Engineering (IBMT), Faculty of Life Science Engineering (LSE), Technische Hochschule Mittelhessen (THM) - University of Applied Sciences, Gießen, Germany, email: melanie.holderith@1se.thm.de

Andreas Rausch, Institute for Biomedical Engineering (IBMT), Faculty of Life Science Engineering (LSE), Technische Hochschule Mittelhessen (THM) - University of Applied Sciences, Gießen, Germany, email: andreas.rausch@1se.thm.de Thomas Schanze, Institute for Biomedical Engineering (IBMT), Faculty of Life Science Engineering (LSE), Technische Hochschule Mittelhessen (THM) - University of Applied Sciences, Gießen, Germany, email: thomas.schanze@1se.thm.de

\section{Introduction}

An electrocardiogram (ECG) measures the heart's electrical activity. The EASI system always uses the same transformation matrices to reconstruct Einthoven and Goldberger leads. No adjustment is made for individual factors such as the patient's age, gender and body type. Thus, an influence of these factors on the reconstruction of the leads is assumed not to exist. The aim of this work is to calculate and examine the transformation matrices for possible dependencies of gender and EASI electrode placement on the reconstruction of Einthoven and Goldberger leads.

\section{Methods}

For this purpose, Einthoven and EASI leads are measured simultaneously on male and female subjects divided into gender-specific classes. The calculation of Goldberger leads was performed subsequently. Based on these data, the transformation matrix was calculated using the Moore-Penrose inverse. Two different methods for calculating the transformation matrix were investigated. Method 1 provides a transformation matrix per class based on individually calculated transformation matrices per subject with subsequent averaging of all individual matrices. In method 2, the individual leads are concatenated to form a common lead, which was then used to calculate a transformation matrix per class. Additionally, the influence of misplaced EASI electrodes on the transformation matrices per class was investigated.

\section{Results}

The results show that it is not possible to reproduce the transformation coefficients reported in the literature with the examined methods and thus comparability cannot be achieved. Furthermore, no similarity of the transformation matrices between the classes could be found. What could be found is an influence on the coefficients when the EASI electrodes are de-placed.

\section{Conclusion}

An important result is that the obtained transformation coefficients only very roughly match with the literature values. The same holds for transformation matrices obtained for the different classes. Thus, using only one generally transformation matrix to reconstruct Einthoven and Goldberger leads from EASI recordings is questionable. An influence of the EASI electrode placement on the matrices could also be demonstrated, so care should be taken to position the EASI electrodes accurately. 


\section{Quantitative assessment of ventricular far field removal techniques for clinical unipolar electrograms}

Nick Johannes Lorenz, Institute of Biomedical Engineering, Karlsruhe Institute of Technology, Karlsruhe, Germany, nick.lorenz@student.kit.edu

Laura Anna Unger, Institute of Biomedical Engineering, Karlsruhe Institute of Technology, Karlsruhe, Germany, laura.unger@kit.edu

Armin Luik, Medizinische Klinik IV, Städtisches Klinikum Karlsruhe, Academic Teaching Hospital of the University of Feiburg, Karlsruhe, Germany, Armin.Luik@klinikum-karlsruhe.de

Olaf Dössel, Institute of Biomedical Engineering, Karlsruhe Institute of Technology, Karlsruhe, Germany, Olaf.Doessel@kit.edu

\section{Introduction}

The incidence of atrial tachycardia steadily increases in industrial nations. During invasive electrophysiological studies, a catheter can measure electrograms within the atrium to assist detailed diagnosis and treatment planning. Unipolar electrograms have several advantages over bipolar electrograms such as the independence from catheter orientation and a higher spatial resolution. However, unipolar electrograms are more affected by noise and the ventricular far field. Therefore, commonly only bipolar electrograms are used in clinical settings. Previously suggested approaches to overcome the disadvantages of unipolar electrograms lead to a prolongation of the electrophysiological study. A recently published ventricular far field removal technique from our group promises a good far field removal, it does not entail a prolongation of the clinical procedure but it lacks quantitative clinical validation.

\section{Methods}

The best performing technique on simulated data approximated the ventricular far field as superposition of electrical potentials emitted by dipoles that were placed in the ventricles. The spatio-temporal model of the ventricular far field was parametrized by previously acquired atrial electrograms. We adapted the dipole method to clinical needs and applied it to two exemplary clinical datasets. Each dataset contained an ultra-high-density electroanatomical map of the left atrium recorded with a 64-pole Orion catheter in sensed paced rhythm. We measured the quality of ventricular far field removal by the distance to a reference signal derived from the baseline within the removal window during sensed paced rhythm using four different lengths of the removal window.

\section{Results}

The ventricular far field model was trained with approximately 120,000 ventricular far field segments per patient. The dipole method resulted in a median reduction of the ventricular far field by approximately $89 \%$ using a removal window of optimal length for both patients. The results showed that the length of the removal window should be selected on a patient-specific basis to account for different expressions and timings of ventricular depolarization and repolarization.

\section{Conclusion}

In conclusion, the dipole method proved suitable for ventricular far field removal in a clinical setting and outperforms comparable methods both in applicability and quality of the results. 


\section{Use of a trained denoising autoencoder to estimate the noise level in the ECG}

Fars Samann, Institut für Biomedizinische Technik (IBMT), FB Life Science Engineering (LSE), Technische Hochschule Mittelhessen (THM) - University of Applied Sciences, Gießen, Germany,

fars.esmat.fathel.samann@1se.thm.de, and, Biomedical Engineering Department, College of Engineering, University of Duhok, Duhok, Kurdistan Region, Iraq

Thomas Schanze, Institut für Biomedizinische Technik (IBMT), FB Life Science Engineering (LSE), Technische Hochschule Mittelhessen (THM) - University of Applied Sciences, Gießen, Germany, thomas.schanze@lse.thm.de

\section{Introduction}

Noise level estimation plays an important role in many signal processing applications, like denoising, compression and detection. In this paper, we develop an efficient method of noise level estimation of ECG segments, based on trained denoising autoencoder (DAE) with a statistical thresholding method.

\section{Methods}

The proposed method is based on training a denoising autoencoder with a single hidden layer with a corrupted training dataset which consist of 300 QRS aligned ECG segments (segment length 80 samples and $f_{s}=100 \mathrm{~Hz}$ ). Then, the same training dataset without any noise is applied to the input of the trained DAE model to estimate the statistical threshold (STH) for clean and aligned ECG segment. This is achieved by calculating the mean and standard deviation of each ECG segment and estimate the p-value for specific probability, e.g., $90 \%$. To evaluate the performance of the noise estimator, a testing dataset of 100 QRS aligned ECG segments is corrupted with different Gaussian white noise levels $(S N R i=5 \mathrm{~dB}, 10 \mathrm{~dB}$ and $15 \mathrm{~dB})$. After applying the testing segment, the Euclidean distance (ED) between the input and the output of the trained DAE model is calculated. If the ED $<$ STH, then the ECG segment is noise free. Otherwise, the ECG segment is noisy. Then, we estimate the noise level by calculating the signal to noise ratio between the input segment and the output segment.

\section{Results}

The accuracy of detecting free noise and noisy ECG segments for $S N R i=5 \mathrm{~dB}, 10 \mathrm{~dB}$ and $15 \mathrm{~dB}$ is $94 \%, 100 \%$, $100 \%$ and $95 \%$ respectively. The standard deviation error of noise estimation in case of $S N R i=5 \mathrm{~dB}, 10 \mathrm{~dB}$ and 15 $\mathrm{dB}$ is $\pm 0.53 \mathrm{~dB}, \pm 0,49 \mathrm{~dB}, \pm 0.78 \mathrm{~dB}$ respectively.

\section{Conclusion}

The proposed method succeeds in classifying noise free and noisy ECG segments and in estimating the noise level with acceptable standard deviation error. 